EPJ Web of Conferences 32, 04006 (2012)

DOI: $10.1051 /$ epjconf/20123204006

(C) Owned by the authors, published by EDP Sciences, 2012

\title{
Large Scale CW ECRH Systems: Some considerations
}

\author{
V. Erckmann ${ }^{1}$, W. Kasparek ${ }^{2}$, B. Plaum ${ }^{2}$, C. Lechte ${ }^{2}$, M.I. Petelin ${ }^{3}$, H. Braune ${ }^{1}$, G. Gantenbein ${ }^{4}$, \\ H. P. Laqua ${ }^{1}$, L.Lubiako ${ }^{3}$, N. B. Marushchenko ${ }^{1}$, G. Michel ${ }^{1}$, Y. Turkin ${ }^{1}$, M. Weissgerber ${ }^{1}$, and the \\ W7-X ECRH- teams at IPP Greifswald ${ }^{1}$, IPF Stuttgart ${ }^{2}$, and KIT $^{4}$

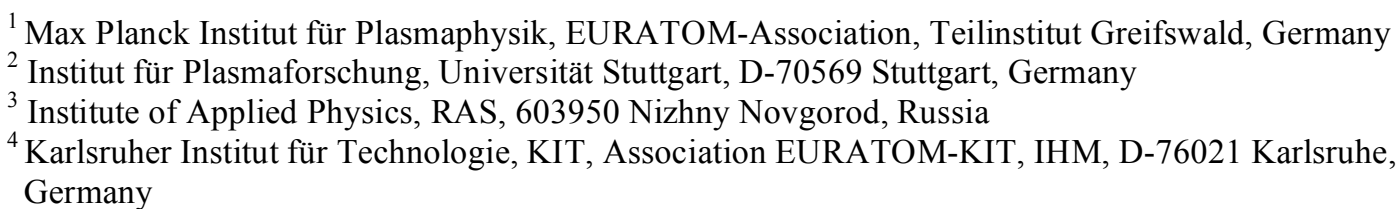

\begin{abstract}
Electron Cyclotron Resonance Heating (ECRH) is a key component in the heating arsenal for the next step fusion devices like W7-X and ITER. These devices are equipped with superconducting coils and are designed to operate steady state. ECRH must thus operate in $\mathrm{CW}$-mode with a large flexibility to comply with various physics demands such as plasma start-up, heating and current drive, as well as configurationand MHD - control. The request for many different sophisticated applications results in a growing complexity, which is in conflict with the request for high availability, reliability, and maintainability. 'Advanced' ECRH-systems must, therefore, comply with both the complex physics demands and operational robustness and reliability. The W7-X ECRH system is the first CW- facility of an ITER relevant size and is used as a test bed for advanced components. Proposals for future developments are presented together with improvements of gyrotrons, transmission components and launchers.
\end{abstract}

\section{Introduction}

Localized and well controllable power deposition as well as simple remote wave launching and beam control is an attractive feature of Electron Cyclotron Resonance Heating (ECRH). The good theoretical understanding of the wave-particle interaction physics, which is benchmarked in many experiments, provides a good quantitative predictability [1]. ECRH complies with various physics demands such as controlled plasma start-up from the neutral gas, steady state plasma stability control, and performance optimization by plasma profile shaping using both the heating and current drive capability. Both, the W7-X Stellarator and the ITER Tokamak rely on powerful CW systems [2,3], which are similar in frequency $(140 \mathrm{GHz}, 10 \mathrm{MW}, 1800 \mathrm{~s}$ for W7-X and $170 \mathrm{GHz}, 24 \mathrm{MW}$, $1000 \mathrm{~s}$ for ITER). Localized current drive (CD) is a key feature for the control of both, the MHD instabilities in Tokamaks and the magnetic configuration in Stellarators.

The request for many different sophisticated applications - some of them are vital for steady state plasma control - and the increasing power demand results in a growing complexity of the systems. This is in conflict with the request for high Reliability, Availability, Maintainability, and Inspectability (RAMI), which arise from DEMO demands. In the case, that reactor operation is based on continuous full power ECRH, the efficiency of every subcomponent has to be maximized 
in order to reduce the recirculating power of the plant. The requirements would be significantly relaxed for reactor concepts, which do not rely on this demand and the ECRH-system can be optimized with respect to economic aspects. 'Advanced' ECRH-components must comply with both the complex physics demands and operational robustness. As present day ECRH-systems have an experimental rather than an industrial character, the achievement of acceptable RAMI standards may require improvements and, very likely, simplifications. The development of refined technological solutions such as advanced gyrotrons, fast high-power beam switches, power combiners [4], and remote-steering launchers must take both aspects into account. Main goals are to extend the operational margins of gyrotrons, to make more efficient use of the installed power, and reduce the requirement for port space. The W7-X ECRH system is the first CW facility of an ITER-relevant size and is used also as a test bed for such new components. Diplexers are versatile transmission components with the capability to replace mechanical switches, improve the efficiency for MHDcontrol techniques, reduce the number of transmission lines and launchers, and share common antennas for ECRH and ECE diagnostics. Remote-steering launchers may experience a revival in view of DEMO, because front-steering launchers with their delicate steering mechanisms facing the burning plasma may not be acceptable. Advanced components for more reliable gyrotron operation are reported in chapter 2. Advanced transmission and launching concepts are discussed in chapter 3 with focus on high-power diplexers and remote steering launchers.

\section{Sources}

The needs of W7-X and ITER were the main drivers for the development of CW gyrotrons at the MW power level with a frequency of $140 \mathrm{GHz}$ (W7-X, 2nd Harmonic at $2.5 \mathrm{~T}$ ) and $170 \mathrm{GHz}$ (ITER, 1 st harmonic at $5.4 \mathrm{~T}$ ). Prototypes of the ITER-gyrotrons were successfully developed in Japan and Russia [5,6]. In EU a more ambitious R\&D aims at 2 MW power per unit [7]. The development of the W7-X gyrotrons was launched in 1998 in EU (THALES) and US (CPI). Both manufacturers demonstrated the specified $1 \mathrm{MW}, \mathrm{CW}$ performance in 2005 [8,9]. A contract for 7 series gyrotrons was then placed with THALES after completion of the first R\&D-milestone in 2003, but only the first series tube, which was delivered in 2005, met the specified parameters. The next series gyrotrons failed to meet the full specifications, although no design changes were made. An ongoing R\&D aiming at the improvement of internal and external gyrotron components to achieve robust industrial series production characterized the following phase. The following components saw a significant improvement since the prototype achievements in 2005:

a) collector sweeping to extend the power margin for the spent electron beam dump

b) isolation of the body voltage

c) window cooling to replace water by oil to prevent long-term corrosion

d) electron beam tunnel to suppress parasitic oscillations

e) shaft coating to reduce stray radiation absorption

A comparison of the TED SN3R gyrotron, which has none of the above mentioned design changes incorporated, and SN4R with improvements a) - d) built in, illustrates the achievements: SN3R is operating very reliably at a $700 \mathrm{~kW}$ power level (27 min tested), whereas operation at higher power leads to unstable RF emission, excitation of parasitic oscillations and interrupts. The TED SN4R gyrotron operates reliably and $1.02 \mathrm{MW}$ were achieved with even some additional power margin. The pulse duration at $>0.9 \mathrm{MW}$ had to be limited, however, to avoid overheating of the stainless steel shaft due to stray radiation absorption. The measured temperature rise of the shaft is about 7 ${ }^{\circ} \mathrm{C} / \mathrm{min}$ and thus the pulse duration was restricted to $1325 \mathrm{~s}$ corresponding to a shaft temperature of $190^{\circ} \mathrm{C}$ (safety limit) at pulse end. As there is no access for active cooling in the narrow gap between the gyrotron shaft and the borehole of the SC- magnet, the inner surface of the shaft of the next series gyrotron SN6 was copper coated to reduce the stray radiation absorption and thus the 
temperature increase. The temperature rise of the shaft was reduced to about $1{ }^{\circ} \mathrm{C} / \mathrm{min}$ at an output power of $920 \mathrm{~kW}$ as expected. This gyrotron showed a robust performance similar to the previous SN4R, but with the capability to safely increase the pulse duration to the specified $1800 \mathrm{~s}$ as shown in Fig. 1 (left).
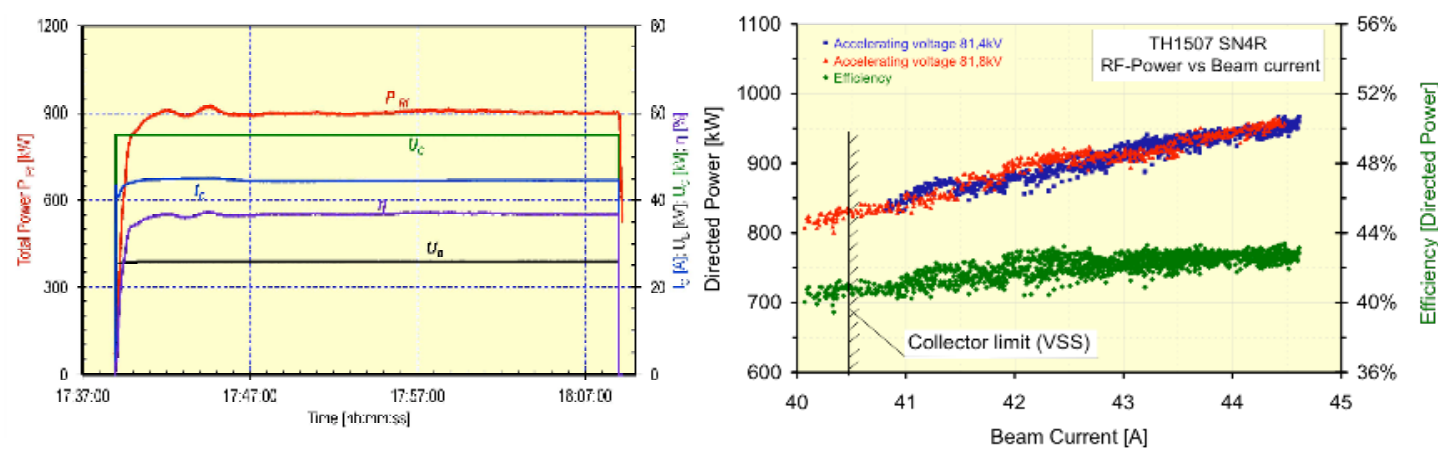

Fig.1: Output power $P_{R F}$, cathode voltage $U_{C}$, beam current $I_{C}$, efficiency $\eta$, and body voltage $U_{B}$ as a function of time for the TED gyrotron SN6 (left). Output power (triangles, squares) and efficiency (diamonds) as a function of beam current for the TED gyrotron SN4R. The limit for the collector loading with a standard vertical sweeping system (VSS) is indicated (right).

The RF power is determined calorimetrically and corrected for the transmission losses through 8 mirrors, the atmospheric attenuation over $18 \mathrm{~m}$, the scattering of higher-order modes in the beam, and the reflection from the load (in total $7 \%$ ). The gyrotron showed no parasitic oscillations in the high-power regime in contrast to SN3R, which is attributed to the redesign of the beam tunnel.

The efficiency of both, the SN4R and the SN6 series gyrotrons is lower than the specified minimum efficiency of $45 \%$, the reasons are subject to further research. The gyrotron collector is designed for a maximum loading of $1.2 \mathrm{MW}$ with power distribution by vertical sweeping. The efficiency of SN6 is typically $40 \%$ and the collector loading thus limits the output power to about $800 \mathrm{~kW}$. The collector loading-limit can be significantly increased by generating a smooth power distribution profile along the z-axis of the collector rather than a profile with strong power peaking at the turning points as generated by conventional vertical sweeping. The peak loading could be reduced to $60 \%$, by application of a combined transverse $(50 \mathrm{~Hz})$ and vertical $(7 \mathrm{~Hz})$ sweeping method [10] and thus a large margin with respect to collector loading was available. Both, the SN4 R and the SN6 gyrotrons were safely operated at a beam current of about $45 \mathrm{~A}$, which is beyond the collector loading limit for the standard vertical collector sweep as seen from Fig.1 (right).

The transition from prototype achievements, where in general the performance is pushed to the very limits, to reproducible series production of units with high reliability and availability is a process with a time scale comparable to the prototype R\&D timescale itself. This statement holds for the W7-X gyrotrons and is supported by experience with earlier R\&D. From the gyrotron tests we conclude also, that operation at, say, $30 \%$ reduced performance, is helpful to arrive at an acceptable reliability. Note, that from the JAERI gyrotrons impressive reliability tests of the 1 MW ITERgyrotrons were reported for operation at reduced power of $800 \mathrm{~kW}$ [11].

For next-step reactor-type devices RAMI aspects will become of crucial importance. A reduction of the diversity of gyrotron types and frequencies and concentration of the R\&D efforts towards a 'standard' type of gyrotron may help to improve the situation. A 3 frequency (3f) standard gyrotron is proposed, which aims at a 1.5 MW design, but is operated at 1.0-1.2 MW for maximum reliability. The chosen triplet of frequencies should be resonant with a simple single-disk window at 
multiples of $\lambda / 2$. The W7-X (TED) and ASDEX Upgrade (AUG) (GYCOM) gyrotrons have diamond windows with a resonant thickness of $4 \lambda / 2$ at $140 \mathrm{GHz}$. They are also transparent at 105 $\mathrm{GHz}$ corresponding to $3 \lambda / 2[10]$, and $175 \mathrm{GHz}$ at $5 \lambda / 2$. A possible set of modes, which are compatible with a generic cavity design is TE22,6 (104 GHz), TE28.8 (140 GHz), and TE34,10 (174 $\mathrm{GHz})[10,11]$, or TE17,6 (104.7 GHz) and TE22,8 (140 GHz) as built from GYCOM for AUG [6]. The development of a $2 \mathrm{f}$ gyrotron at JAEA is based on TE31,11 (170 GHz) and TE25.9 (137 GHz), both frequencies match the resonances of a single disk window with $1.853 \mathrm{~mm}$ thickness [12]. The chosen modes are both emitted from the mode converter at about the same inclination angle and can thus be coupled to the transmission waveguide by the same MOU. About 1.3 MW output power was achieved at both frequencies. Tuning the resonant magnetic field and adjusting the operation parameters can excite the different modes. An additional advantage can be taken from a generic magnet design, which should, under RAMI aspects, be cryogen-free and capable of handling the required magnetic configurations for all three modes and frequencies. As seen from table 1, where the ECRH-frequencies of some fusion devices are listed, the basic needs of these devices may be satisfied within some acceptable margin by gyrotrons generating a frequency triple of 175/140/105 GHz or, anchored at the ITER-frequency, at 170/137/102 GHz. The needs of DIII-D and TCV would not fit into this frame.

Table 1. ECRH frequencies of a $3 f$ gyrotron and the frequency demand of some fusion devices.

\begin{tabular}{|l|c|c|c|c|c|c|c|c|}
\hline & ITER & W7-X & JT60SA & $\begin{array}{c}\text { ASDEX- } \\
\text { UPGRADE }\end{array}$ & EAST & KSTAR & DIII-D & TCV \\
\hline $\mathbf{1 0 2 / 1 0 5}$ & & 105 & 110 & 105 & & & $110 / 117.5$ & 118 \\
\hline $\mathbf{1 3 7 / 1 4 0}$ & & 140 & 138 & 140 & 140 & & & \\
\hline $\mathbf{1 7 0 / 1 7 5}$ & 170 & & & & & 170 & & \\
\hline
\end{tabular}

The present day gyrotrons are designed as oscillators. In combination with interferometer types of high-power diplexers (see next section) it would be advantageous, however, to operate phase locked gyrotrons or gyro-amplifiers, which can be phase controlled by a master generator. Encouraging short-pulse results were obtained with a gyro-klystron operating at $30 \mathrm{GHz}$ in TE5,3 mode with 15 MW output power at $30 \%$ efficiency [13].

\section{Transmission and launching}

\subsection{Four-port diplexers as TL-switches and beam combiners}

ECRH systems are composed of many units of typically 1-2 MW power, where each unit consists of a gyrotron, an attributed transmission line (TL) and launcher channel. The physics demands as outlined before, require rf-power switches to direct the source power to different TL's and launchers. In the ITER baseline design, mechanical waveguide switches are foreseen in every TL to switch the power between the equatorial Heating- and CD-launcher and the upper launchers, which are dedicated to MHD-control. Mechanical switches are, however, slow and the rf-power has to be turned off during switching. The implementation of high power, four-port diplexers as nonmechanical fast directional switches (FADIS) avoids these drawbacks and allows toggling of the power from a continuously operating gyrotron between two transmission lines or launchers. Switching is effected by either tuning the gyrotron frequency between slightly different frequencies $f_{1}$ and $f_{2}$ as sketched in Fig. 2 (left), or tuning the optical length of the resonator. High power experiments on fast switching up to $20 \mathrm{kHz}$ were performed by a small frequency shift keying of the gyrotron(s) by modulation of the gun anode or the beam acceleration voltage [4]. Diplexers can also be used to combine the RF beams from two (or more) gyrotrons as sketched in Fig. 2 (middle) thus reducing the number of transmission lines and launchers [4]. Installing diplexers at a proper location in the TL's, they can provide fast directional switching and, at the same time provide the option for a power upgrade by feeding an additional gyrotron to the second input (see Fig. 2 (left)). Note, that for 
ITER a power upgrade in a later phase of the experiment is being discussed and would be possible without adding TL's, ports and launchers. As the minimization of port space may become a major issue under DEMO-aspects, resonant diplexers are of interest also for plasma diagnostics because the same TL can be used for power transmission and sensitive ECE detection as sketched in Fig. 2 (right).

For the ECE-measurement in MHD-stabilization experiments, the "Line-of-Sight" scheme [14] exploits the gyrotron transmission line in the reverse direction, thus ensuring that the ECE observed originates from the deposition region of ECRH. Note, that the sophisticated fast calculation of plasma profiles and related 'real-time' equilibrium reconstruction for position feed back becomes obsolete for this type of observation and may simplify the MHD-control feed back system.
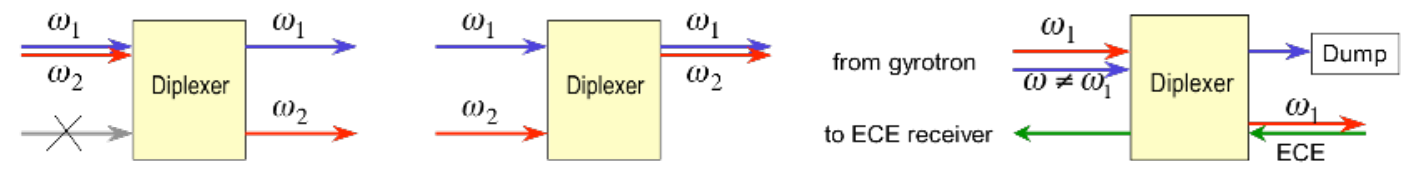

Fig.2: Examples for diplexer application (schematic): FADIS (left), Beam Combiner (middle), and In-line ECE (right).

The transmission characteristics of diplexers can be tailored according to the needs and several types were investigated in low power experiments [15]. Driven by the physics demands for advanced NTM-control experiments at AUG, a resonant diplexer with a narrow resonance of about $12 \mathrm{MHz}$ FWHM and a free spectral range of $141 \mathrm{MHz}$ was chosen. These design features are particularly well suited for FADIS, because of the steep resonant slope, for power combination, because two gyrotrons operating at slightly different frequency of the order of half the free spectral range can be combined to one output channel, and, last not least, for in-line ECE, because it provides good isolation from the power channel. The device was equipped with a feedback controlled movable mirror as tuning element [16], which matches the transmission characteristics to variations of the gyrotron frequency. Tuning the mirror by typically half a wavelength causes switching between the two outputs, which is interesting for applications in the ms-time scale, where no gyrotron-tuning is needed, or a variable splitting ratio of the power is envisaged. The resonant diplexer under investigation was installed into a straight corrugated waveguide section of the transmission system at AUG after successful high power tests using the W7-X ECRH. First preparatory experiments on MHD-control and in-line ECE have started [17,18].

\subsection{Launchers}

Launchers have to be optimized taking into account the gyrotron features: For standard gyrotons with fixed frequency the launchers must cope with the demands on power localization in the plasma (poloidal launch angle) and current drive (toroidal launch angle), or both. For step-tuneable gyrotrons, where the power deposition region is selected by the frequency, the launcher functionality can be restricted to toroidal (horizontal) motion only to adjust the current drive. Under RAMI aspects the simplification of the launcher mechanism is, however, counteracted by the gyrotron complexity in the latter case. In both, the W7-X and ITER devices, the ECRH power is fed to the plasma through quasi-optical front-steering launchers. These launchers provide a wide angular steering range and a narrow focusing of the individual RF beams. In ITER the wide steering range is required for MHD stabilization at $\mathrm{q}=1,1.5,2$, whereas for $\mathrm{W} 7-\mathrm{X}$ the envisaged high-density $\mathrm{H} \& \mathrm{CD}$ scenarios with $\mathrm{O}$ $\mathrm{X}-\mathrm{B}$ mode conversion heating are accessible only with strong oblique launch. The narrow power deposition is needed in ITER for MHD control, which is most efficient for complete power deposition within the narrow magnetic islands at the O-point. For W7-X the narrow deposition is 
favourable, because the minor radius of the plasma is small $(\mathrm{a}=0.55 \mathrm{~m})$. The drawback of front steering is the complicated steering mechanism inside the vacuum vessel and a comparatively large coupling structure with the related port space. For the W7-X and the ITER Upper Launcher, a similar averaged power density (port through power/ port area) of 8-11 $\mathrm{MWm}^{-2}$ (baseline design) or twice this number assuming $2 \mathrm{MW} / \mathrm{beam}$, is obtained. Under RAMI aspects it is expected, however, that a front steering launcher in the vicinity of the burning plasma is incompatible with DEMO demands. Thus the remote steering (RSL) concept may experience a revival, although some compromise with respect to steering range and focusing has to be made. The plasma facing, in-vessel structure is a simple corrugated square waveguide with no moving parts. The beam steering is performed out of the vessel. The average power density of such a simple structure would be about $100 \mathrm{MWm}^{-2}$, i.e. one (small) port would be sufficient to transmit the complete H\&CD power for DEMO. Possible improvement of RSLs are the extension of the steering range, development of low-loss, high power $\mathrm{CW}$ structures $(2 \mathrm{MW})$, mode conserving mitre bends with arbitrary angle, integration of vacuum valves and barrier windows. For W7-X we have started conceptual design work on improved, CWcompatible, RSLs for the high field side launch. The RSL consists of a basically square corrugated waveguide with cross-section about $50 \times 50 \mathrm{~mm}$, however, with some modification to enlarge the steering range $[19,20]$. The integration between the coupling mirror situated in the main transmission system and the $\mathrm{N}$-port of $\mathrm{W} 7-\mathrm{X}$ requires a mitre bend halfway along the waveguide. The waveguide will be evacuated, with the vacuum barrier window installed directly at the entrance of the waveguide. This avoids vignetting of the beam at larger steering angles; however, it requires that the vacuum valve be situated within the waveguide run. Extensive calculations have been performed to optimize the various parameters of the RSL. A corrugation profile with rounded edges and large aspect ratio has been calculated to minimize the ohmic loss in the wall. The investigations are expected to prepare a solid basis for the design of RSL's for next step devices.

\section{References}

[1] V. Erckmann, Fusion Engineering and Design, 84 (2009) 131-137

[2] M. Shimada et al., Nucl. Fusion 47 (2007) S1-S17

[3] V. Erckmann et al., Fusion Science and Technology, Vol. 52, No.2 (2007) pp. 291-312

[4] W. Kasparek, et al. Nucl. Fusion 48 (2008) 05401

[5] K. Sakamoto, A. Kasugai, K. Takahashi, et al. Nature physics, Vol. 3, June 2007

[6] A.G. Litvak, G.G. Denisov, et al., J. Infrared Milli\& TeraHz Waves (2011) 32: 337-342

[7] B. Piosczyk, et al. IEEE Trans. Plasma Sci. 32 (2004) 413

[8] K. Felch, M. Blank, et al., Journal of Physics: Conference Series 25 (2005) pp. 13-23.

[9] M. Thumm, P. Brand, et al., IEEE Trans. Plasma Science, Vol. 36, No.2 (2008) 341-355

[10] V. Erckmann, P. Brand, et al., AIP Conf. Proc. Vol. 933: RF Power in Plasmas (2007) 421-424

[11] S. Kern et al., IRMMW-THz 2008. 10.1109/ICIMW.2008.4665582

[12] K. Sakamoto, K. Kajiwara, Y. Oda, et al., 978-1-4577-0509-0/11/\$26.00 (2011) IEEE

[13] M. Petelin et al., Proc. 11th IEEE Int. Vacuum Electr. Conf., Monterey, CA, 2010, 191 - 192

[14] W. Bongers et al., Fusion Sci. Technol. 55 (2009) 188-203

[15] A. Bruschi, V. Erckmann, et al. IEEE Trans. Plasma Science, Vol. 38, No. 6 (2010) 1427-1438

[16] N. Doelmann, et al., this conference

[17] W. Bongers, W. Kasparek, et al., this conference

[18] J. Stober, M. Reich, et al., this conference

[19] G.G. Denisov, K.A. Fedorova, et al., Int. J. Infrared Milli Waves (2007) 28:923-935

[20] B. Plaum, E. Holzhauer, and C. Lechte,. to be published 